OPEN ACCESS

Edited by:

Gilles Gouspillou,

Université du Québec à Montréal,

Canada

Reviewed by:

Ann Marie Zavacki,

Brigham and Women's Hospital, USA

Damien Roussel,

Université Claude Bernard Lyon 1

France

*Correspondence:

Assunta Lombardi,

Department of Biology, University of

Naples Federico II, Via Cinthia

Complesso Universitario MSA

(Edificio 7), 80126 Naples, Italy

assunta.Iombardi@unina.it:

Fernando Goglia,

Department of Science and

Technology, University of Sannio, Via

Port'Arsa 11, 82100 Benevento, Italy

goglia@unisannio.it

${ }^{\dagger}$ These authors have contributed equally to this work.

Specialty section: This article was submitted to

Striated Muscle Physiology, a section of the journal

Frontiers in Physiology

Received: 26 June 2015 Accepted: 07 August 2015 Published: 21 August 2015

Citation: Lombardi A, Moreno M, de Lange $P$, lossa S, Busiello RA and Goglia F (2015) Regulation of skeletal muscle mitochondrial activity by thyroid hormones: focus on the "old" triiodothyronine and the "emerging" 3,5-diiodothyronine. Front. Physiol. 6:237.

doi: 10.3389/fphys.2015.00237

\section{Regulation of skeletal muscle} mitochondrial activity by thyroid hormones: focus on the "old" triiodothyronine and the "emerging" 3,5-diiodothyronine

\author{
Assunta Lombardi ${ }^{1 * t}$, Maria Moreno ${ }^{2 t}$, Pieter de Lange ${ }^{3}$, Susanna lossa ${ }^{1}$, \\ Rosa A. Busiello ${ }^{2}$ and Fernando Goglia ${ }^{2 *}$ \\ ${ }^{1}$ Department of Biology, University of Naples Federico II, Naples, Italy, ${ }^{2}$ Department of Science and Technology, University of \\ Sannio, Benevento, Italy, ${ }^{3}$ Department of Environmental, Biological and Pharmaceutical Sciences and Technologies, Second \\ University of Naples, Caserta, Italy
}

3,5,3'-Triiodo-L-thyronine (T3) plays a crucial role in regulating metabolic rate and fuel oxidation; however, the mechanisms by which it affects whole-body energy metabolism are still not completely understood. Skeletal muscle (SKM) plays a relevant role in energy metabolism and responds to thyroid state by remodeling the metabolic characteristics and cytoarchitecture of myocytes. These processes are coordinated with changes in mitochondrial content, bioenergetics, substrate oxidation rate, and oxidative phosphorylation efficiency. Recent data indicate that "emerging" iodothyronines have biological activity. Among these, 3,5-diiodo-L-thyronine (T2) affects energy metabolism, SKM substrate utilization, and mitochondrial functionality. The effects it exerts on SKM mitochondria involve more aspects of mitochondrial bioenergetics; among these, respiratory chain activity, mitochondrial thermogenesis, and lipid-handling are stimulated rapidly. This mini review focuses on signaling and biochemical pathways activated by T3 and T2 in SKM that influence the above processes. These novel aspects of thyroid physiology could reveal new perspectives for understanding the involvement of SKM mitochondria in hypo- and hyper-thyroidism.

Keywords: mitochondria, lipid metabolism, uncoupling, thyroid hormones, diiodothyronines

\section{Introduction}

Skeletal muscle (SKM) is a metabolically active tissue representing about $40 \%$ of total body mass. It significantly affects energy expenditure and plays a significant role in glucose, lipid, and energy homeostasis. SKM shows a remarkable plasticity in functional adaptation and remodeling in response to physiological stimuli, such as exercise, fasting, and hormonal signals. Among the hormones able to influence SKM development, metabolism, and structure, thyroid hormone (T3) plays a key role (Salvatore et al., 2014). SKM responds to variations in thyroid state by coordinately remodeling its cytoarchitecture and metabolic characteristics, with mitochondria playing a significant role. Concerning metabolic adaptations, T3 enhances the use of lipids and carbohydrates as fuel substrates (de Lange et al., 2008; Lombardi et al., 2012), as well as alteration of mitochondrial number and functionality. 
Growing evidence indicates 3,5-diiodo-L-thyronine (T2) as a biologically active thyroid hormone derivative able to affect energy metabolism (Goglia, 2015 and references within). T2 increases resting metabolic rate, enhances lipid utilization as a fuel substrate, and prevents the occurrence of dietinduced obesity and associated diseases, including liver steatosis, hypertriglyceridemia, hypercholesterolemia (Lanni et al., 2005; de Lange et al., 2011), and insulin resistance (de Lange et al., 2011; Moreno et al., 2011). In SKM, T2 ameliorates the tissue's response to insulin that is impaired by a high fat diet (Moreno et al., 2011). Importantly, previous studies have shown that, contrary to T3, T2 does not induce thyrotoxicity or undesirable side effects at the cardiovascular level at the doses used $(25 \mu \mathrm{g} / 100 \mathrm{~g}$ rat body weight, Lanni et al., 2005; de Lange et al., 2011).

The present mini review provides an overview of the involvement of SKM mitochondria in T3 and/or T2 effects exerted on modulation of SKM metabolism/plasticity. In particular, it focuses on signaling and biochemical pathways activated by the two iodothyronines in SKM, promoting variations in substrate metabolism, lipid handling, and thermogenesis at the mitochondrial level.

\section{Effect of Thyroid Hormones on SKM Mitochondrial Biogenesis}

T3 influences mitochondrial activity and biogenesis by modulating, in a coordinate fashion, expression of proteins encoded by both the nuclear and mitochondrial genome.

\section{Nuclear Events}

T3 acts through nuclear receptors (TRs), namely TR $\alpha$ and TR $\beta$, ligand-dependent transcription factors that are constitutively bound to thyroid hormone response elements (TREs). The binding of T3 to TRs leads to stimulation or inhibition of nuclear gene transcription (Brent, 2012). T3 regulates the transcription of a series of genes harboring TREs (direct T3 target genes), some of which serve as intermediate factors (e.g., transcriptional factors and coactivators) needed to regulate a second series of genes (indirect T3 target genes). In SKM, T3-modulated transcription is primarily mediated by the $\mathrm{TR} \alpha 1$ isoform and involves a wide array of genes influencing SKM contractile and metabolic properties, as well as those coding components of the tricarboxylic acid cycle and mitochondrial respiratory chain (Wiesner et al., 1992; Short et al., 2001; Clement et al., 2002). In SKM, T3 also influences the transcription of genes controlling mRNA maturation and protein translation. Indeed, T3 upregulates transcripts encoding ribunucleoproteins and splicing factors as well as ribosomal proteins and translation initiation factors (eIF1A, Clement et al., 2002).

T3 positively regulates the expression of intermediate factors, such as nuclear respiratory factors (NRF)-1 and -2, which enhance the expression of mitochondrial transcription factor-A, a nuclear-encoded transcription factor essential for replication, maintenance, and transcription of mitochondrial DNA. T3 also controls the expression of coactivator of peroxisome proliferator activated receptor $\gamma$ (PPAR $\gamma$ ) PGC-1 $\alpha$ (Weitzel et al., 2001), a central regulator of mitochondrial gene expression and biogenesis (Puigserver, 2005). PGC-1 $\alpha$ regulates gene expression through its interactions with DNA-bound transcription factors, including TR, PPAR, and NRF-1 (Knutti and Kralli, 2001, Figure 1).

In rats, the effect induced by $\mathrm{T} 3$ on mitochondrial content and activity is amplified in slow oxidative compared to fast glycolytic muscles (Bahi et al., 2005). This could be explained by higher expression of TR $\alpha 1$ and PGC1 $\alpha$ observed in slow oxidative muscle (Garnier et al., 2003; Bahi et al., 2005) associated with opposite regulation of $\mathrm{TR} \alpha$ transcription by $\mathrm{T} 3$ in the two distinct muscle types (activation in slow oxidative and reduction in fast glycolytic muscle). Conversely, in humans, T3 does not influence NRF-1 or PGC1 $\alpha$ levels in SKM (Barbe et al., 2001). Thus, the effect of T3 on SKM mitochondrial biogenesis seems to be species-specific and dependent on SKM metabolism.

In SKM, AMP-activated kinase (AMPK) regulates the expression of genes related to mitochondrial biogenesis, energy production, and oxidative protection. AMPK phosphorylates and activates PGC1 $\alpha$ (Jäger et al., 2007; Cantó et al., 2009), and both chronic and acute administration of T3 to euthyroid (Irrcher et al., 2008) and hypothyroid rats (Branvold et al., 2008; de Lange et al., 2008) induces AMPK activation, a putative mediator of the effect of T3 on SKM mitochondrial biogenesis (Figure 1).

\section{Mitochondrial Events}

Mitochondrial gene expression is also directly activated by T3 through its binding to mitochondrial matrix-localized specific receptors (p43). p43 is a truncated form of TR $\alpha 1$ and is synthesized by the use of an internal initiation site of translation (Wrutniak et al., 1995; Wrutniak-Cabello et al., 2001). The T3-p43 complex binds to TREs of the mitochondrial genome and induces transcription (Casas et al., 1999) in parallel to the transcription of nuclear genes involved in oxidative phosphorylation, thus ensuring complementary signaling between nuclear and mitochondrial pathways (Figure 1). p43 regulates SKM phenotypes, contractile features, and metabolism. In mice, p43 deletion leads to muscle hypertrophy and a shift in the direction of more rapid muscle fiber types coordinated with a reduction in mitochondrial content (Pessemesse et al., 2012). p43 overexpression leads to muscle wasting with aging (Casas et al., 2009), suggesting a possible oxidative stress-associated toxic effect due to prolonged stimulation of mitochondrial activity, leading to a deficit of new skeletal muscle fiber replacement and differentiation over time.

P28 is another truncated form of TR- $\alpha 1$ that is localized in the inner mitochondrial membrane and shows a binding affinity for T3 higher than nuclear receptors. Despite it has been shown that $\mathrm{p} 28$ regulates mitochondrial functionality in fibroblast, its specific function has not been elucidated yet (Pessemesse et al., 2014).

\section{Effect of T2 on Mitochondrial Biogenesis}

Studies supporting the possibility of T2's effects on mitochondrial biogenesis have been focused on liver and brown adipose tissue (de Lange et al., 2011; Lombardi et al., 2015). In SKM, it is possible that activation of AMPK by T2 (Lombardi et al., 2009a) could trigger transcriptional processes leading to mitochondrial 


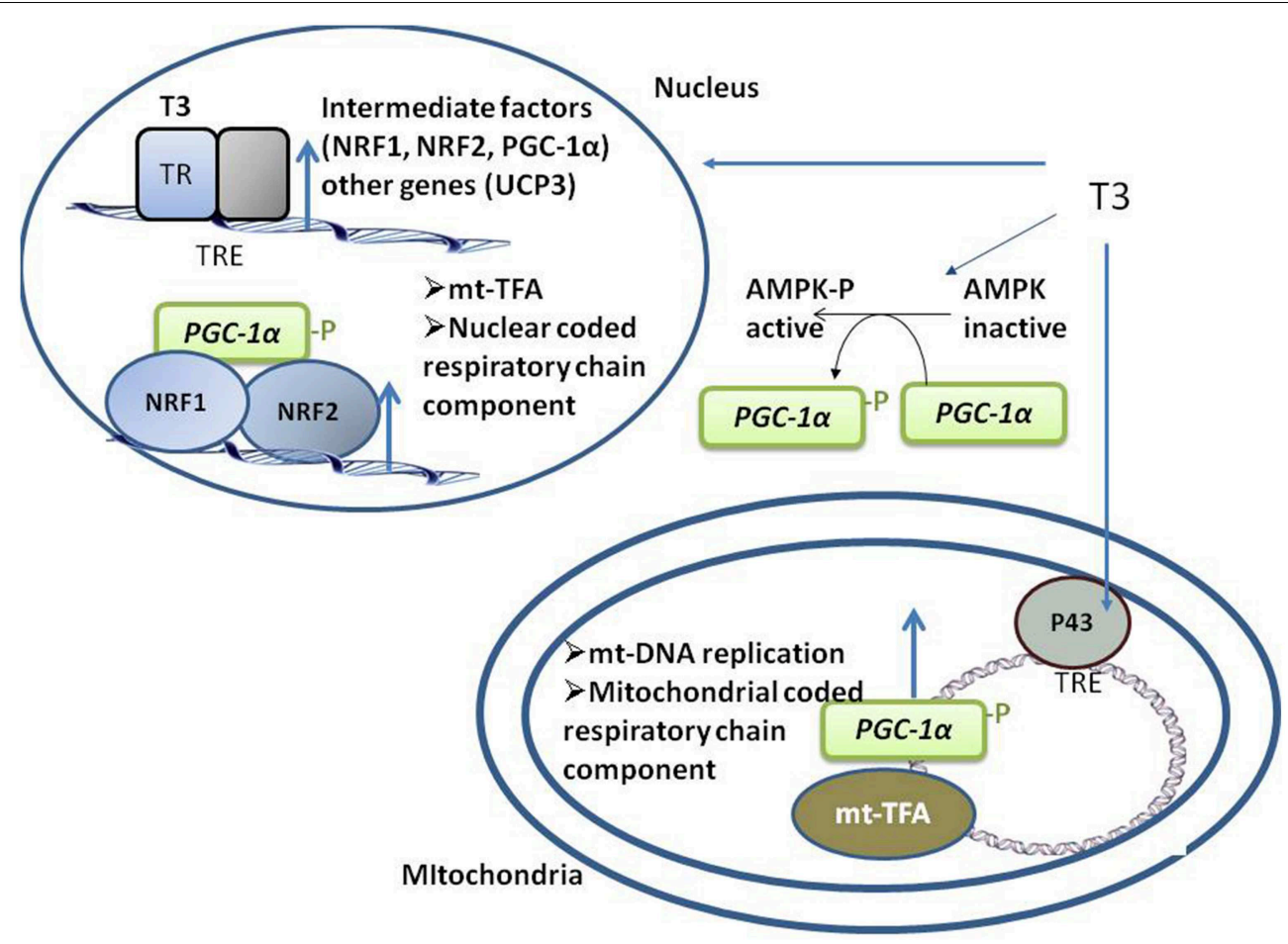

FIGURE 1 | Schematic representation of the coordinated events activated by T3 that take place in both nuclei and mitochondria, promoting mitochondrial biogenesis. T3 directly activates the transcription of nuclear- and mitochondrial-genes coding for component of respiratory chain by binding to its nuclear TRs and mitochondrial p43. T3 also indirectly activates respiratory genes transcription by up-regulating the transcription of intermediate factors (such as NRF-1 and -2 and PGC1a). AMPK activation also mediates an indirect effect of T3 on mitochondrial biogenesis. biogenesis (see above). Direct evidence regarding the ability of T2 to influence mitochondrial biogenesis in SKM is currently lacking and this aspect needs further investigation.

Whether or not the effects of T2 are mediated by TRs is still under investigation. A recent study in mice showed that $\mathrm{T} 2$ evokes TR-mediated effects only when administered at very high doses $(250 \mu \mathrm{g} / 100 \mathrm{~g}$ bw administered daily for 4 week, Jonas et al., 2015). When used at high doses an interaction of T2 with TRs could take place despite the much lower affinity of T2 for TRs when compared to T3 (Ball et al., 1997; Cioffi et al., 2010; de Lange et al., 2011; Mendoza et al., 2013). Interestingly, in a teleost fish species (Tilapia) T2 interacts with a TR $\beta$ receptor isoform and activates gene transcription ex vivo in a cell- and promoter-specific manner (Mendoza et al., 2013). Thus, further experiments are needed to elucidate whether and how T2 can modulate gene transcription.

\section{Thyroid Hormones Influence SKM Mitochondrial Functionality and Thermogenesis}

Mitochondrial functionality is profoundly affected by thyroid state. In SKM, in the transition between hypo- and hyperthyroidism, a progressive increase in mitochondrial substrate oxidation is detected regardless of substrate [i.e., glycolytic-
(Venditti et al., 2003, 2007) or lipid-associated substrates (Silvestri et al., 2005; Lombardi et al., 2012)].

\section{Mitochondrial Uncoupling}

Mitochondrial respiration is not fully coupled to ATP synthesis since part of the energy contained in the reduced substrate is lost as heat. Most of the uncoupling is due to a leak of protons across the mitochondrial inner membrane (proton-leak); a failure in proton pumping during electron transport (redox slip) also induces mitochondrial uncoupling. In SKM, protonleak accounts for a significant portion of the cellular metabolic rate, either when muscle is at rest (Rolfe and Brand, 1996) or in the contracting state (Rolfe et al., 1999). Proton-leak is the sum of two processes: basal and inducible proton-leak (Brand and Esteves, 2005). Basal proton-leak is not acutely regulated. It depends on the fatty-acyl composition of the mitochondrial inner membrane and on the presence of adenine nucleotide translocase (ANT). Inducible proton-leak is acutely controlled by activation of specific proteins, with uncoupling protein (UCP3 in SKM) and ANT (ANT1 in SKM) playing a crucial role (Divakaruni and Brand, 2011).

\section{T3 Induces SKM Mitochondrial Uncoupling}

SKM mitochondrial uncoupling induced by T3 has been reported in vivo (Lebon et al., 2001) and ex vivo (Lanni et al., 1999; de Lange et al., 2001; Lombardi et al., 2002, 2012). Interestingly, 
despite uncoupling activation by $\mathrm{T} 3$, no variation or increase in SKM ATP levels takes place (Jucker et al., 2000; Lebon et al., 2001; Short et al., 2001). The uncoupling associated with T3-induced mitochondrial biogenesis could counteract possible ATP variations. In addition, an increase in the ability of SKM mitochondria to produce ATP could also take place, as already observed in liver (Harper and Brand, 1993; Nogueira et al., 2002).

The existence of a positive correlation between T3 and SKM mitochondrial proton-leak is also evident during aging. In fact, aging is associated with a decrease in circulating T3 that is evident in 24 month-old rats (Iossa et al., 2002; Silvestri et al., 2008; Valle et al., 2008); therefore, aging represents a condition of physiological hypothyroidism. Concomitantly, SKM mitochondria from old rats exhibited a significant decrease in proton-leak (Lombardi et al., 2009a; Crescenzo et al., 2014), suggesting that with increasing age, the efficiency of oxidative phosphorylation increases in SKM mitochondria. Similar results have been obtained in vivo in aged rat SKM, where a trend of higher coupling efficiency was found (Gouspillou et al., 2014). When mitochondria are more efficient, fewer substrates are oxidized to obtain ATP. Therefore, increased mitochondrial coupling in SKM could contribute to the decreased energy expenditure that characterizes the progression of aging and hypothyroidism since SKM energy metabolism accounts for about $30 \%$ of whole-body energy expenditure under resting conditions (Rolfe and Brown, 1997).

\section{Factors Involved in T3 Induced- SKM Mitochondrial Uncoupling}

T3 affects both basal (Lombardi et al., 2012) and inducible SKM proton-leak (Lanni et al., 1999; Silvestri et al., 2005; Lombardi et al., 2012), with UCP3 and ANT being involved in the effects on free fatty acid (FA)-inducible proton-leak (Figure 2). In the transition between hypo- and hyper-thyroidism, the contribution of ANT to FA-induced uncoupling becomes progressively more relevant despite there being no variation in ANT-1 mRNA levels detected (Dümmler et al., 1996; Lombardi et al., 2002). This could be attributed to the gradual increase in mitochondrial SKM FA levels (Lombardi et al., 2002), known activators of ANT-mediated uncoupling (Skulachev, 1991). Concerning UCP3, T3 increases its transcription (Lanni et al., 1999; Barbe et al., 2001), the effect being observed within $8 \mathrm{~h}$ of T3 administration to hypothyroid rats (de Lange et al., 2001, 2007). The mechanism of UCP3 promoter stimulation by $\mathrm{T} 3$ seems to be species-specific since it involves FA and their target receptors (PPAR $\delta$ ) in humans and rats but not mice (de Lange et al., 2007).

Besides regulating UCP3 expression, T3 also promotes UCP3mediated uncoupling by synergistically stimulating biochemical pathways underlying activation of this protein (Silvestri et al., 2005). Indeed, T3 enhances reactive oxygen species formation (Venditti et al., 2003, 2007; Silvestri et al., 2005) and mitochondrial FA availability (Lombardi et al., 2002; Silvestri et al., 2005) that have been shown to act in combination to induce UCP3-mediated uncoupling (Echtay et al., 2002; Lombardi et al., 2008, 2010). A single administration of T3 to hypothyroid rats induces parallel increases in (i) whole animal resting metabolic rate, (ii) SKM mitochondrial UCP3 content, and (iii) SKM mitochondrial uncoupling, thus indicating the importance of UCP3 in the regulation of rat resting metabolic rate by $\mathrm{T} 3$ (de Lange et al., 2001; Flandin et al., 2009). UCP3 is also involved in mitigation of reactive oxygen species production (Brand and Esteves, 2005) and counteracting lipotoxicity induced by accumulation of FA and lipid hydroperoxides in the mitochondrial matrix (Goglia and Skulachev, 2005; Schrauwen et al., 2006; Lombardi et al., 2010). Thus, the upregulation of UCP3 by T3 would alleviate mitochondrial damage resulting from chronic mitochondrial activation associated with hyperthyroidism.

\section{3,5-T2 Affects Mitochondrial Oxidative Phosphorylation in SKM}

The administration of T2 to hypothyroid rats rapidly enhances both coupled and uncoupled respiration with mechanisms that are independent of de novo transcription and translation (Lombardi et al., 2007). Indeed, T2 promotes activation of the kinetics of the reactions involved in the oxidation of substrates (among these respiratory chain), while not primarily influencing reactions involved in the synthesis and export of ATP (Lombardi et al., 2007, Figure 2).

The mechanism by which T2 affects uncoupled mitochondrial respiration mainly involves proton leak, since $\mathrm{T} 2$ does not affect redox slip nor induce any significant change in the overall respiratory chain $\mathrm{H}^{+} / \mathrm{O}$ ratio (Lombardi et al., 2007). Contrary to what is observed for T3, T2 does not activate basal proton-leak, rather its effect is totally dependent on FA presence (Lombardi et al., 2009a, 2012). Although, it is clear that T2 promotes FAinducible proton-leak, the molecular component involved have not been individuated yet (Figure 2).

\section{Thyroid Hormones Influence SKM Mitochondrial Lipid Handling}

Alterations in thyroid state are associated with changes in energy demand, with SKM adapting its metabolism by modulating substrate utilization. In the hypothyroid state, SKM enhances FA import into myocytes, a process associated with a decrease in the ability of mitochondria to use FA as fuel and enhancement of oxidative phosphorylation efficiency. Thus, the imbalance between FA supply and oxidation leads to accumulation of intramyocyte triglycerides (Lombardi et al., 2012). On the other hand, in the hyperthyroid condition, an increase in FA uptake into myocytes is associated with a rise in FA oxidation, which becomes less efficient because of proton-leak activation. Consequently, FAs are not deposited as triglycerides (Lombardi et al., 2012, Figure 2).

\section{T3 Affects Mitochondrial Fatty Acid Oxidation}

More processes are crucial for SKM mitochondrial FA oxidation, which include FA availability to mitochondria, import of acylCoA into the mitochondrion, mitochondrial oxidative capacity, and feedback inhibition by intermediates present in the FA oxidation pathway. T3 influences all the cited processes. Indeed, it promotes mitochondrial localization of FAT/CD36 (Lombardi et al., 2012), known to increase FA supply to the mitochondria (Holloway et al., 2009). This event is coordinated with the import of acyl-CoA into the mitochondria, obtained by activation of 


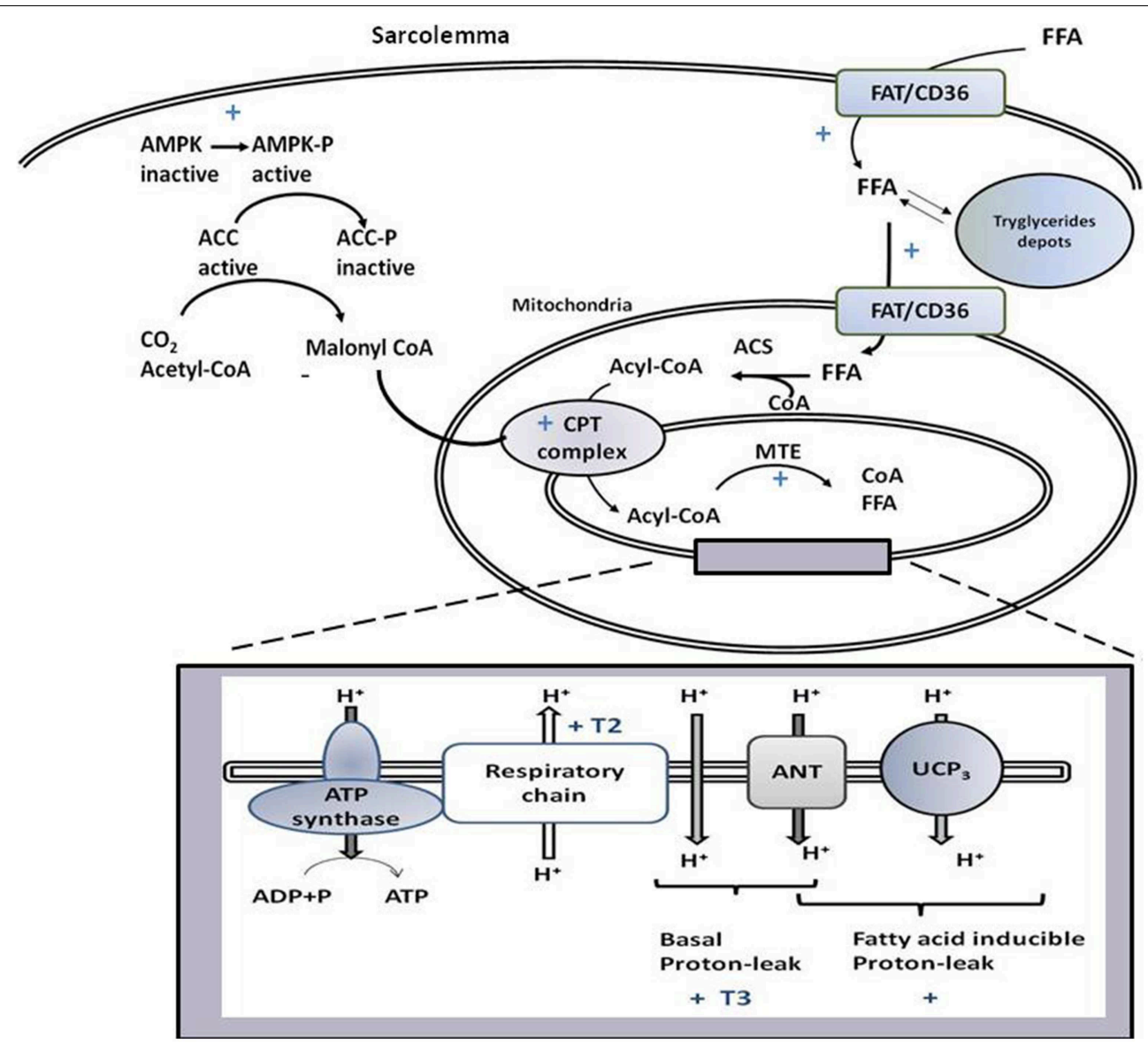

FIGURE 2 | Schematic representation of signaling and biochemical pathways activated by T3 and/or T2 in skeletal muscle that promote variations in substrate metabolism, lipid handling, and thermogenesis at the mitochondrial level. If not accompanied by T3 or $\mathrm{T} 2$, the symbol + indicates that the pathway is stimulated by both iodothyronines. T3 and T2 activate processes leading to the import of FFA and their oxidation at the mitochondrial level, with FAT/CD36 playing a role. Through activation of AMPK-ACC signaling pathway, T2 and T3 relieves the inhibition of CPT1 by malonyl-CoA, and thus promote the entrance of fatty acids into mitochondria and their oxidation. The rise in MTE activity, would contribute to maintain a high level of fatty acid oxidation rate. The box represents processes, occurring at the level of mitochondrial inner membrane, underlying coupled and uncoupled respiration affected by T3 and T2. the carnitine-palmitoyl-transferase (CPT) complex (considered a rate-limiting step for $\mathrm{FA}$ uptake into mitochondrion). T3 modulates transcription of CPT complex components (e.g., CPT-1 and -2, Silvestri et al., 2005). In addition, in SKM, T3 promotes AMPK activation and inhibition of its downstream target, acetyl-CoA carboxylase (ACC, Branvold et al., 2008; de Lange et al., 2008; Irrcher et al., 2008). ACC inhibition leads to a reduction in malonyl-CoA levels that inhibits CPT-1 activity. Thus, the activation of AMPK-ACC-malonyl-CoA signaling leads to sequential enhancement of CPT-1 activity, mitochondrial acyl-CoA uptake, and oxidation (de Lange et al., 2008, Figure 2).

\section{Mitochondrial Lipid Handling and Uncoupling: Interrelated Role in Mediating the Effect of T3 on SKM Mitochondria}

Inside mitochondria, a rise in $\mathrm{NADH} / \mathrm{NAD}^{+}$and $\mathrm{CoA}-$ $\mathrm{SH} /$ acetyl-CoA ratios, as well as accumulation of $\beta$-oxidation intermediate metabolites, can cause feedback inhibition of the $\beta$-oxidation pathway (Koves et al., 2008). The T3induced uncoupling effect contributes to maintaining the above ratios at low levels and thus, functioning to sustain an elevated mitochondrial FA oxidation rate. Furthermore, the activation of SKM intra-mitochondrial thioesterase (MTE; catalyzes cleavage of acyl-CoA to $\mathrm{CoA}$ and free FA) by T3 (Silvestri et al., 2005) contributes to sustaining a high FA oxidation rate, since it maintains a high CoA/acetyl$\mathrm{CoA}$ ratio and supplies $\mathrm{CoA}$, whose pool is limited, for $\beta$ oxidation. Intra-mitochondrial production of FA, catalyzed by MTE, could play a role in FA-induced proton leak activated by T3. Thus, an interlink between lipid-handling and mitochondrial uncoupling coexists: the activation of uncoupling could facilitate the FA oxidation rate and, at the same time, the increase availability of FA to mitochondria, associated with lipid handling, would promote FA-induced mitochondrial uncoupling. 


\section{T2 Affects SKM Fatty Acid Oxidation}

The rapid stimulatory effect of $\mathrm{T} 2$ on mitochondrial respiration seems to be specific to FA metabolism, since T2 does not influence mitochondrial ability to use pyruvate as a substrate (Lombardi et al., 2009a). T2 has an effect similar to that induced by $\mathrm{T} 3$ in increasing SKM FA uptake and channeling FAs to mitochondria and increasing their oxidation. Indeed, T2 and T3 activate translocation of FAT/CD36 from cellular depots to the sarcolemma and mitochondria, each in a very rapid fashion. In this aspect, the two iodothyronines seem to mimic the effect of physical exercise, which influences FAT/CD36-mediated transport of lipids across the sarcolemmal membrane and into the mitochondria (Holloway et al., 2009).

Although both T2 and T3 increase the SKM mitochondrial FA oxidation rate in hypothyroid rats, the onset of CPT and mitochondrial respiratory pathway activation differ since the two processes were already activated $1 \mathrm{~h}$ after T2 administration,

\section{References}

Bahi, L., Garnier, A., Fortin, D., Serrurier, B., Veksler, V., Bigard, A. X., et al. (2005). Differential effects of thyroid hormones on energy metabolism of rat slow- and fast-twitch muscles. J. Cell Physiol. 203, 589-598. doi: 10.1002/ jcp. 20273

Ball, S. G., Sokolov, J., and Chin, W. W. (1997). 3,5-diiodo-L-thyronine (T2) has selective thyromimetic effects in vivo and in vitro. J. Mol. Endocrinol. 19, 137-147. doi: 10.1677/jme.0.0190137

Barbe, P., Larrouy, D., Boulanger, C., Chevillotte, E., Viguerie, N., Thalamas, C., et al. (2001). Triiodothyronine-mediated up-regulation of UCP2 and UCP3 mRNA expression in human SKM without coordinated induction of mitochondrial respiratory chain genes. FASEB J. 15, 13-15. doi: 10.1096/fj.000502fje

Brand, M. D., and Esteves, T. C. (2005). Physiological functions of the mitochondrial uncoupling proteins UCP2 and UCP3. Cell Metab. 2, 85-93. doi: 10.1016/j.cmet.2005.06.002

Branvold, D. J., Allred, D. R., Beckstead, D. J., Kim, H. J., Fillmore, N., Condon, B. M., et al. (2008). Thyroid hormone effects on LKB1, MO25, phosphoAMPK, phospho-CREB, and PGC-1alpha in rat muscle. J. Appl. Physiol. 105, 1218-1227. doi: 10.1152/japplphysiol.00997.2007

Brent, G. A. (2012). Mechanisms of thyroid hormone action. J. Clin. Invest. 122, 3035-3043. doi: 10.1172/JCI60047

Cantó, C., Gerhart-Hines, Z., Feige, J. N., Lagouge, M., Noriega, L., Milne, J. C., et al. (2009). AMPK regulates energy expenditure by modulating NAD+ metabolism and SIRT1 activity. Nature 458, 1056-1060. doi: $10.1038 /$ nature 07813

Casas, F., Pessemesse, L., Grandemange, S., Seyer, P., Baris, O., Gueguen, N., et al. (2009). Overexpression of the mitochondrial T3 receptor induces SKM atrophy during aging. PLOS ONE 4:e5631. doi: 10.1371/journal.pone. 0005631

Casas, F., Rochard, P., Rodier, A., Cassar-Malek, I., Marchal-Victorion, S., Wiesner, R. J., et al. (1999). A variant form of the nuclear triiodothyronine receptor cErbAalphal plays a direct role in regulation of mitochondrial RNA synthesis. Mol. Cell. Biol. 19, 7913-7924.

Cioffi, F., Zambad, S. P., Chhipa, L., Senese, R., Busiello, R. A., Tuli, D., et al. (2010). TRC150094, a novel functional analog of iodothyronines, reduces adiposity by increasing energy expenditure and fatty acid oxidation in rats receiving a high-fat diet. FASEB J. 24, 3451-3461. doi: 10.1096/fj.10-157115

Clement, K., Viguerie, N., Diehn, M., Alizadeh, A., Barbe, P., Thalamas, C., et al. (2002). In vivo regulation of human SKM gene expression by thyroid hormone. Genome Res. 12, 281-291. doi: 10.1101/gr.207702

Crescenzo, R., Bianco, F., Mazzoli, A., Giacco, A., Liverini, G., and Iossa, S. (2014). Alterations in proton leak, oxidative status and uncoupling protein 3 content in whereas T3 was ineffective at that time point (Lombardi et al., 2012). Within $1 \mathrm{~h}, \mathrm{~T} 2$ rapidly activated the AMPK-CPT-malonylCoA signaling pathway that leads to enhancement of FA uptake in mitochondria via increased CPT-1 activity (Figure 2). Rapid activation of MTE-1 and proton-leak by T2 would contribute to maintaining high FA oxidation rates (Lombardi et al., 2009b).

\section{Conclusions}

SKM mitochondrial physiology is profoundly affected by the thyroid state and underlies a significant part of the metabolic effects induced by T3. The recent discovery of T2 as a metabolically active thyroid hormone derivatives indicates that thyroid physiology is continually evolving. These novel aspects of thyroid physiology could reveal new perspectives for understanding the contribution of SKM mitochondria to different thyroid states. skeletal muscle subsarcolemmal and intermyofibrillar mitochondria in old rats. BMC Geriatr. 4:79. doi: 10.1186/1471-2318-14-79

de Lange, P., Cioffi, F., Senese, R., Moreno, M., Lombardi, A., Silvestri, E., et al. (2011). Nonthyrotoxic prevention of diet-induced insulin resistance by 3,5-diiodo-L-thyronine in rats. Diabetes 60, 2730-2739. doi: 10.2337/ db11-0207

de Lange, P., Feola, A., Ragni, M., Senese, R., Moreno, M., Lombardi, A., et al. (2007). Differential 3,5,3'-triiodothyronine-mediated regulation of uncoupling protein 3 transcription: role of Fatty acids. Endocrinology 148, 4064-4072. doi: 10.1210/en.2007-0206

de Lange, P., Lanni, A., Beneduce, L., Moreno, M., Lombardi, A., Silvestri, E., et al. (2001). Uncoupling protein-3 is a molecular determinant for the regulation of resting metabolic rate by thyroid hormone. Endocrinology 142, 3414-3420. doi: 10.1210/endo.142.8.8303

de Lange, P., Senese, R., Cioffi, F., Moreno, M., Lombardi, A., Silvestri, E., et al. (2008). Rapid activation by 3,5,3'-L-triiodothyronine of adenosine 5 '-monophosphate-activated protein kinase/acetyl-coenzyme a carboxylase and akt/protein kinase B signaling pathways: relation to changes in fuel metabolism and myosin heavy-chain protein content in rat gastrocnemius muscle in vivo. Endocrinology 149, 6462-6470. doi: 10.1210/ en.2008-0202

Divakaruni, A. S., and Brand, M. D. (2011). The regulation and physiology of mitochondrial proton-leak. Physiology 26, 192-205. doi: 10.1152/physiol.00046.2010

Dümmler, K., Müller, S., and Seitz, H. J. (1996). Regulation of adenine nucleotide translocase and glycerol 3-phosphate dehydrogenase expression by thyroid hormones in different rat tissues. Biochem. J. 317, 913-918.

Echtay, K. S., Roussel, D., St-Pierre, J., Jekabsons, M. B., Cadenas, S., Stuart, J. A., et al. (2002). Superoxide activates mitochondrial uncoupling proteins. Nature 415, 96-99. doi: 10.1038/415096a

Flandin, P., Lehr, L., Asensio, C., Giacobino, J. P., Rohner-Jeanrenaud, F., Muzzin, P., et al. (2009). Uncoupling protein-3 as a molecular determinant of the action of 3,5,3'-triiodothyronine on energy metabolism. Endocrine 36, 246-254. doi: 10.1007/s12020-009-9217-8

Garnier, A., Fortin, D., Delomenie, C., Momken, I., Veksler, V., and VenturaClapier, R. (2003). Depressed mitochondrial transcription factors and oxidative capacity in rat failing cardiac and SKMs. J. Physiol. 551, 491-501. doi: 10.1113/jphysiol.2003.045104

Goglia, F., and Skulachev, V. P. (2005). A function for novel uncoupling proteins: antioxidant defense of mitochondrial matrix by translocating fatty acid peroxides from the inner to the outer membrane leale. FASEB J. 17, 1585-1591. doi: 10.1096/fj.03-0159hyp

Goglia, F. (2015). The effects of 3,5-diiodothyronine on energy balance. Front. Physiol. 5:528. doi: 10.3389/fphys.2014.00528 
Gouspillou, G., Bourdel-Marchasson, I., Rouland, R., Calmettes, G., Biran, M., Deschodt-Arsac, V., et al. (2014). Mitochondrial energetics is impaired in vivo in aged skeletal muscle. Aging Cell 13, 39-48. doi: 10.1111/acel.12147

Harper, M. E., and Brand, M. D. (1993). The quantitative contributions of mitochondrial proton leak and ATP turnover reactions to the changed respiration rates of hepatocytes from rats of different thyroid status. J. Biol. Chem. 268, 14850-14860.

Holloway, G. P., Jain, S. S., Bezaire, V., Han, X. X., Glatz, J. F., Luiken, J. J., et al. (2009). FAT/CD36-null mice reveal that mitochondrial FAT/CD36 is required to upregulate mitochondrial fatty acid oxidation in contracting muscle. Am. J. Physiol. Regul. Integr. Comp. Physiol. 297, R960-R967. doi: 10.1152/ajpregu.91021.2008

Iossa, S., Mollica, M. P., Lionetti, L., Crescenzo, R., Botta, M., Barletta, A., et al. (2002). Acetyl-carnitine supplementation differently influences nutrient partitioning, serum leptin concentration and skeletal muscle mitochondrial respiration in young and old rats. J. Nutr. 132, 636-642.

Irrcher, I., Walkinshaw, D. R., Sheehan, T. E., and Hood, D. A. (2008). Thyroid hormone (T3) rapidly activates p38 and AMPK in skeletal muscle in vivo. J. Appl. Physiol. 104, 178-185. doi: 10.1152/japplphysiol.00643.2007

Jäger, S., Handschin, C., St-Pierre, J., and Spiegelman, B. M. (2007). AMPactivated protein kinase (AMPK) action in skeletal muscle via direct phosphorylation of PGC-1alpha. Proc. Natl. Acad. Sci. U.S.A. 104, 12017-1222. doi: 10.1073/pnas.0705070104

Jonas, W., Lietzow, J., Wohlgemuth, F., Hoefig, C. S., Wiedmer, P., Schweizer, U., et al. (2015). 3,5-diiodo-L-thyronine (3,5- $\left.\mathrm{T}_{2}\right)$ exerts thyromimetic effects on hypothalamus-pituitary-thyroid axis, body composition, and energy metabolism in male diet-induced obese mice. Endocrinology 156, 389-399. doi: 10.1210/en.2014-1604

Jucker, B. M., Dufour, S., Ren, J., Cao, X., Previs, S. F., Underhill, B., et al. (2000). Assessment of mitochondrial energy coupling in vivo by 13C/31P NMR. Proc. Natl. Acad. Sci. U.S.A. 97, 6880-6884. doi: 10.1073/pnas.120131997

Knutti, D., and Kralli, A. (2001). PGC-1, a versatile coactivator. Trends Endocrinol. Metab. 12, 360-365. doi: 10.1016/S1043-2760(01)00457-X

Koves, T. R., Ussher, J. R., Noland, R. C., Slentz, D., Mosedale, M., Ilkayeva, O., et al. (2008). Mitochondrial overload and incomplete fatty acid oxidation contribute to skeletal muscle insulin resistance. Cell Metab. 7, 45-56. doi: 10.1016/j.cmet.2007.10.013

Lanni, A., Beneduce, L., Lombardi, A., Moreno, M., Boss, O., Muzzin, P., et al. (1999). Expression of uncoupling protein-3 and mitochondrial activity in the transition from hypothyroid to hyperthyroid state in rat skeletal muscle. FEBS Lett. 444, 250-254.

Lanni, A., Moreno, M., Lombardi, A., de Lange, P., Silvestri, E., Ragni, M., et al. (2005). 3,5-diiodo-L-thyronine powerfully reduces adiposity in rats by increasing the burning of fats. FASEB J. 19, 1552-1554. doi: 10.1096/fj.053977 fje

Lebon, V., Dufour, S., Petersen, K. F., Ren, J., Jucker, B. M., Slezak, L. A., et al. (2001). Effect of triiodothyronine on mitochondrial energy coupling in human skeletal muscle. J. Clin. Invest. 108, 733-737. doi: 10.1172/JCI11775

Lombardi, A., Busiello, R. A., Napolitano, L., Cioffi, F., Moreno, M., de Lange, P., et al. (2010). UCP3 translocates lipid hydroperoxide and mediates lipid hydroperoxide-dependent mitochondrial uncoupling. J. Biol. Chem. 285, 16599-16605. doi: 10.1074/jbc.M110.102699

Lombardi, A., de Lange, P., Silvestri, E., Busiello, R. A., Lanni, A., Goglia, F., et al. (2009a). 3,5-diiodo-L-thyronine rapidly enhances mitochondrial fatty acid oxidation rate and thermogenesis in rat skeletal muscle: AMP-activated protein kinase involvement. Am. J. Physiol. Endocrinol. Metab. 296, E497-E502. doi: 10.1152/ajpendo.90642.2008

Lombardi, A., De Matteis, R., Moreno, M., Napolitano, L., Busiello, R. A., Senese, R., et al. (2012). Responses of skeletal muscle lipid metabolism in rat gastrocnemius to hypothyroidism and iodothyronine administration: a putative role for FAT/CD36. Am. J. Physiol. Endocrinol. Metab. 303, E1222-E1233. doi: 10.1152/ajpendo.00037.2012

Lombardi, A., Grasso, P., Moreno, M., de Lange, P., Silvestri, E., Lanni, A., et al. (2008). Interrelated influence of superoxides and free fatty acids over mitochondrial uncoupling in skeletal muscle. Biochim. Biophys. Acta 1777, 826-833. doi: 10.1016/j.bbabio.2008.04.019

Lombardi, A., Lanni, A., de Lange, P., Silvestri, E., Grasso, P., Senese, R., et al. (2007). Acute administration of 3,5-diiodo-L-thyronine to hypothyroid rats affects bioenergetic parameters in rat skeletal muscle mitochondria. FEBS Lett. 581, 5911-5916. doi: 10.1016/j.febslet.2007. 11.073

Lombardi, A., Senese, R., De Matteis, R., Busiello, R. A., Cioffi, F., Goglia, F., et al. (2015). 3,5-diiodo-L-thyronine activates brown adipose tissue thermogenesis in hypothyroid rats. PLoS ONE 10:e0116498. doi: 10.1371/journal.pone.0116498

Lombardi, A., Silvestri, E., Cioffi, F., Senese, R., Lanni, A., Goglia, F., et al. (2009b). Defining the transcriptomic and proteomic profiles of rat ageing skeletal muscle by the use of a cDNA array, 2D- and Blue native-PAGE approach. J. Proteomics 72, 708-721. doi: 10.1016/j.jprot.2009.02.007

Lombardi, A., Silvestri, E., Moreno, M., de Lange, P., Farina, P., Goglia, F., et al. (2002). Skeletal muscle mitochondrial free-fatty-acid content and membrane potential sensitivity in different thyroid states: involvement of uncoupling protein-3 and adenine nucleotide translocase. FEBS Lett. 532, 12-16. doi: 10.1016/S0014-5793(02)03690-6

Mendoza, A., Navarrete-Ramírez, P., Hernández-Puga, G., Villalobos, P., Holze, G., Renaud, J. P., et al. (2013). 3,5- $\mathrm{T}_{2}$ is an alternative ligand for the thyroid hormone receptor $\beta 1$. Endocrinology 154, 2948-2958. doi: 10.1210/en.20131030

Moreno, M., Silvestri, E., De Matteis, R., de Lange, P., Lombardi, A., Glinni, D., et al. (2011). 3,5-diiodo-L-thyronine prevents high-fat-diet-induced insulin resistance in rat skeletal muscle through metabolic and structural adaptations. FASEB J. 25, 3312-3324. doi: 10.1096/fj.11-181982

Nogueira, V., Walter, L., Avéret, N., Fontaine, E., Rigoulet, M., and Leverve, X. M. (2002). Thyroid status is a key regulator of both flux and efficiency of oxidative phosphorylation in rat hepatocytes. J. Bioenerg. Biomembr. 34, 55-66 doi: 10.1023/A:1013822820840

Pessemesse, L., Lepourry, L., Bouton, K., Levin, J., Cabello, G., Wrutniak-Cabello, C., et al. (2014). p28, a truncated form of TR $\alpha 1$ regulates mitochondrial physiology. FEBS Lett. 588, 4037-4043. doi: 10.1016/j.febslet.2014.09.026

Pessemesse, L., Schlernitzauer, A., Sar, C., Levin, J., Grandemange, S., Seyerv, P., et al. (2012). Depletion of the p43 mitochondrial T3 receptor in mice affects SKM development and activity. FASEB J. 26, 748-756. doi: 10.1096/fj.11195933

Puigserver, P. (2005). Tissue-specific regulation of metabolic pathways through the transcriptional coactivator PGC1-alpha. Int. J. Obes. (Lond.) 29, S5-S9. doi: 10.1038/sj.ijo.0802905

Rolfe, D. F., and Brand, M. D. (1996). Proton-leak and control of oxidative phosphorylation in perfused, resting rat SKM. Biochim. Biophys. Acta 1276, 45-50. doi: 10.1016/0005-2728(96)00029-1

Rolfe, D. F., Newman, J. M., Buckingham, J. A., Clark, M. G., and Brand, M. D. (1999). Contribution of mitochondrial proton-leak to respiration rate in working SKM and liver to SMR. Am. J. Physiol. 276(Pt 1), C692-C699.

Rolfe, D. F. S., and Brown, G. C. (1997). Cellular energy utilisation and molecular origin of standard metabolic rate in mammals. Physiol. Rev. 77, 731-758.

Salvatore, D., Simonides, W. S., Dentice, M., Zavacki, A. M., and Larsen, P. R. (2014). Thyroid hormones and skeletal muscle-new insights and potential implications. Nat. Rev. Endocrinol. 10, 206-214. doi: 10.1038/ nrendo.2013.238

Schrauwen, P., Hoeks, J., and Hesselink, M. K. (2006). Putative function and physiological relevance of the mitochondrial uncoupling protein-3: involvement in fatty acid metabolism? Prog. Lipid Res. 45, 17-41. doi: 10.1016/j.plipres.2005.11.001

Short, K. R., Nygren, J., Barazzoni, R., Levine, J., and Nair, K. S. (2001). $\mathrm{T}(3)$ increases mitochondrial ATP production in oxidative muscle despite increased expression of UCP2 and -3. Am. J. Physiol. Endocrinol. Metab. 280, E761-E769.

Silvestri, E., Lombardi, A., de Lange, P., Schiavo, L., Lanni, A., Goglia, F., et al. (2008). Age-related changes in renal and hepatic cellular mechanisms associated with variations in rat serum thyroid hormone levels. Am. J. Physiol. Endocrinol. Metab. 294, E1160-E1168. doi: 10.1152/ajpendo.00044.2008

Silvestri, E., Moreno, M., Lombardi, A., Ragni, M., de Lange, P., Alexson, S. E., et al. (2005). Thyroid-hormone effects on putative biochemical pathways involved in UCP3 activation in rat skeletal muscle mitochondria. FEBS Lett. 579, 1639-1645. doi: 10.1016/j.febslet.2005.02.022

Skulachev, V. P. (1991). Fatty acid circuit as a physiological mechanism of uncoupling of oxidative phosphorylation. FEBS Lett. 294, 158-162. doi: 10.1016/0014-5793(91)80658-P 
Valle, A., Santandreu, F. M., García-Palmer, F. J., Roca, P., and Oliver, J. (2008). The serum levels of $17 \beta$-estradiol, progesterone and triiodothyronine correlate with brown adipose tissue thermogenic parameters during aging. Cell Physiol. Biochem. 22, 337-346. doi: 10.1159/000149812

Venditti, P., Bari, A., Di Stefano, L., and Di Meo, S. (2007). Role of mitochondria in exercise-induced oxidative stress in skeletal muscle from hyperthyroid rats. Arch. Biochem. Biophys. 463, 12-18. doi: 10.1016/j.abb.2007. 02.004

Venditti, P., Puca, A., and Di Meo, S. (2003). Effect of thyroid state on rate and sites of $\mathrm{H} 2 \mathrm{O} 2$ production in rat skeletal muscle mitochondria. Arch. Biochem. Biophys. 411, 121-128. doi: 10.1016/S0003-9861(02)00720-8

Weitzel, J. M., Radtke, C., and Seitz, H. J. (2001). Two thyroid hormone-mediated gene expression patterns in vivo identified by cDNA expression arrays in rat. Nucleic Acids Res. 29, 5148-5155. doi: 10.1093/nar/29.24.5148

Wiesner, R. J., Kurowski, T. T., and Zak, R. (1992). Regulation by thyroid hormone of nuclear and mitochondrial genes encoding subunits of cytochrome-c oxidase in rat liver and SKM. Mol. Endocrinol. 6, 1458-1467.
Wrutniak, C., Cassar-Malek, I., Marchal, S., Rascle, A., Heusser, S., Keller, J. M., et al. (1995). A $43-\mathrm{kDa}$ protein related to c-Erb A alpha 1 is located in the mitochondrial matrix of rat liver. J. Biol. Chem. 270, 16347-16354.

Wrutniak-Cabello, C., Casas, F., and Cabello, G. (2001). Thyroid hormone action in mitochondria. J. Mol. Endocrinol. 26, 67-77. doi: 10.1677/jme.0.0260067

Conflict of Interest Statement: The authors declare that the research was conducted in the absence of any commercial or financial relationships that could be construed as a potential conflict of interest.

Copyright $\odot 2015$ Lombardi, Moreno, de Lange, Iossa, Busiello and Goglia. This is an open-access article distributed under the terms of the Creative Commons Attribution License (CC BY). The use, distribution or reproduction in other forums is permitted, provided the original author(s) or licensor are credited and that the original publication in this journal is cited, in accordance with accepted academic practice. No use, distribution or reproduction is permitted which does not comply with these terms. 